PROCEEDINGS OF THE

AMERICAN MATHEMATICAL SOCIETY

Volume 128, Number 5, Pages 1451-1457

S 0002-9939(99)05323-X

Article electronically published on December 8, 1999

\title{
A WILD MINIMAL PLANE IN $\mathbb{R}^{3}$
}

\author{
PLÁCIDO ANDRADE
}

(Communicated by Peter Li)

\begin{abstract}
The main object of this article is to construct a complete minimal immersed plane in $\mathbb{R}^{3}$ whose closure has nonempty interior but it is not dense in the whole space. Furthermore, its Gaussian curvature is bounded.
\end{abstract}

\section{INTRODUCTION}

In [Rsb], Rosenberg contructed a dense minimal surface in $\mathbb{R}^{3}$ by reflection of a fundamental domain. For this he used a result of Jenkins-Serrin [J-S]. Inspired by Rosenberg's example, Jorge [Jor] asked whether a complete minimal surface in $\mathbb{R}^{3}$ with bounded Gaussian curvature is either proper or dense. The answer is negative. The purpose of this note is to construct a complete minimal immersed surface in $\mathbb{R}^{3}$ which is conformal to a plane and whose closure has nonempty interior. Moreover it is not dense in the whole space and the Gaussian curvature is bounded. For this purpose we will need the following result from [And]. Here, we identify $\mathbb{R}^{3}$ with $\mathbb{C} \times \mathbb{R}$.

Theorem 1. Let $h$ be a harmonic function in an open set $\Omega \subset \mathbb{C}$. Suppose that $L$ and $H$ are holomorphic functions in $\Omega$ such that

$$
L^{\prime} H^{\prime}=\left(\frac{\partial h}{\partial z}\right)^{2} \quad \text { and } \quad\left|L^{\prime}(z)\right|+\left|H^{\prime}(z)\right|>0,
$$

for $z \in \Omega$. Then the mapping

$$
\chi: \Omega \rightarrow \mathbb{C} \times \mathbb{R}, \quad \chi(z)=(L(z)-\overline{H(z)}, h(z))
$$

is a conformal minimal immersion.

We call a map $\chi$ satisfying equation (1) an Enneper immersion. In [And], we prove this result and show that any immersed minimal surface in $\mathbb{C} \times \mathbb{R}$ can be parametrized by an Enneper immersion.

Theorem 2. There exists a complete immersed minimal plane in $\mathbb{C} \times \mathbb{R}$ whose closure contains the xy-plane in its interior. Furthermore, it is not dense in the whole space and its Gaussian curvature is bounded.

To show the existence of that plane we consider a dense curve in a cylindrical shell of revolution and solve the Plateau problem for it. The author wishes to thanks his colleague at UFC, L. Jorge, for clarifying conversations.

Received by the editors June 23, 1998.

1991 Mathematics Subject Classification. Primary 53A10; Secondary 53C42.

(C)2000 American Mathematical Society 
The paper is organized into three sections. Section 1 is this introduction. For sake of completeness, in Section 2 we present the proof of Theorem 1. In Section 3 we construct the example.

\section{Proof of Theorem 1}

We will use the standard inner product; given $u=\left(a_{1}+i b_{1}, c_{1}\right)$ and $v=$ $\left(a_{2}+i b_{2}, c_{2}\right) \in \mathbb{C} \times \mathbb{R}$ we define

$$
\langle u, v\rangle=a_{1} a_{2}+b_{1} b_{2}+c_{1} c_{2} .
$$

Lemma 1. If $\chi: \Omega \rightarrow \mathbb{C} \times \mathbb{R}, \chi(z)=(a(z)+i b(z), c(z))$, is a $C^{1}$ map, then

$$
(E-G)-2 i F=4\left[\left(\frac{\partial a}{\partial z}\right)^{2}+\left(\frac{\partial b}{\partial z}\right)^{2}+\left(\frac{\partial c}{\partial z}\right)^{2}\right],
$$

where $E=\left\langle\chi_{u}, \chi_{u}\right\rangle, G=\left\langle\chi_{v}, \chi_{v}\right\rangle$ and $F=\left\langle\chi_{u}, \chi_{v}\right\rangle$.

Proof. A straightforward computation shows that

$$
(E-G)-2 i F=\left(a_{u}-i a_{v}\right)^{2}+\left(b_{u}-i b_{v}\right)^{2}+\left(c_{u}-i c_{v}\right)^{2} .
$$

Since

$$
a_{u}-i a_{v}=2 \frac{\partial a}{\partial z}, \quad b_{u}-i b_{v}=2 \frac{\partial b}{\partial z} \quad \text { and } \quad c_{u}-i c_{v}=2 \frac{\partial c}{\partial z},
$$

we easily complete the proof of the lemma.

Proof (Theorem 1). Assume that $\chi(z)=(L(z)-\overline{H(z)}, h(z))$ is a map such that the functions $L(z)=p(z)+i q(z)$ and $H(z)=f(z)+i g(z)$ are holomorphic and $h$ is harmonic. The Cauchy-Riemann equations allow us to write the derivatives of $L$ and $H$ as

$$
\frac{1}{2} L^{\prime}=\frac{\partial p}{\partial z}=i \frac{\partial q}{\partial z} \quad \text { and } \quad \frac{1}{2} H^{\prime}=\frac{\partial f}{\partial z}=i \frac{\partial g}{\partial z} .
$$

Since $\chi=((p-f)+i(q+g), h)$, from Lemma 1, we may write the identities

$$
\begin{aligned}
(E-G)-2 i F & =4\left[\left(\frac{\partial(p-f)}{\partial z}\right)^{2}+\left(\frac{\partial(q+g)}{\partial z}\right)^{2}+\left(\frac{\partial h}{\partial z}\right)^{2}\right] \\
& =4\left[\frac{1}{4}\left(L^{\prime}-H^{\prime}\right)^{2}+\frac{1}{4}\left(-i L^{\prime}-i H^{\prime}\right)^{2}+\left(\frac{\partial h}{\partial z}\right)^{2}\right] \\
& =4\left[-L^{\prime} H^{\prime}+\left(\frac{\partial h}{\partial z}\right)^{2}\right] .
\end{aligned}
$$

From now on, let us suppose that the functions $L, H$ and $h$ satisfy the differential equation (1.1). This implies that $E=G$ and $F=0$. Since $\left\|\chi_{u}\right\|=\left\|\chi_{v}\right\|$, to prove that $\chi$ is a conformal immersion it is sufficient to show that the partial derivative $\chi_{u}$ does not vanish. Indeed, assume that $\chi_{u}\left(z_{0}\right)=(0,0,0)=\chi_{v}\left(z_{0}\right)$ for some point $z_{0} \in \Omega$; in other words, assume that

$$
p_{u}\left(z_{0}\right)-f_{u}\left(z_{0}\right)=0, \quad q_{u}\left(z_{0}\right)+g_{u}\left(z_{0}\right)=0 \quad \text { and } \quad h_{u}\left(z_{0}\right)=0=h_{v}\left(z_{0}\right) .
$$

It is immediate to verify that $H^{\prime}\left(z_{0}\right)=\overline{L^{\prime}\left(z_{0}\right)}$ and $h_{u}\left(z_{0}\right)=0$. From (1.1), it follows that $4\left|L^{\prime}\left(z_{0}\right)\right|^{2}=0$. Thus $\left|L^{\prime}\left(z_{0}\right)\right|=0=\left|H^{\prime}\left(z_{0}\right)\right|$, which is a contradiction because $\left|L^{\prime}(z)\right|+\left|H^{\prime}(z)\right| \neq 0$ for any $z \in \Omega$. So, $\chi$ is a conformal immersion. It still 
remains to be shown that $\chi$ is minimal. Let $N$ be the unit vector field normal to the immersion. Since the mean curvature $\mathcal{H}$ is given by

$$
\mathcal{H}=\frac{G l+E n-2 F m}{2\left(E G-F^{2}\right)}
$$

where $l=\left\langle N, \chi_{u u}\right\rangle, n=\left\langle N, \chi_{v v}\right\rangle$ and $m=\left\langle N, \chi_{u v}\right\rangle$ and since each coordinate of $\chi$ is a harmonic function we have $\chi_{u u}=-\chi_{v v}$, which implies that $l=-n$. With those conditions, it is easy to verify that the mean curvature is zero. This completes the proof of Theorem 1.

The Weierstrass data of $\chi$ can be easily obtained from the usual formulas. Following the notations of $[\mathrm{B}-\mathrm{C}]$, we have

$$
(S, g, w)=\left(\Omega,-\frac{L^{\prime}}{\frac{\partial h}{\partial z}}, 2 \frac{\partial h}{\partial z} d z\right) .
$$

The first fundamental form and the Gauss curvature induced on $\Omega$ are, respectively,

$$
d s=\left(\left|L^{\prime}\right|+\left|H^{\prime}\right|\right)|d z| \quad \text { and } \quad K=-4\left[\frac{\left|\frac{\partial h}{\partial z}\right|\left|\frac{d g}{g}\right|}{\left(\left|L^{\prime}\right|+\left|H^{\prime}\right|\right)^{2}}\right]^{2} \text {. }
$$

\section{The EXAMPle}

Let $d, r_{1}, r_{2}$ be fixed real numbers with $r_{2} \neq 0$. We will call trochoid a parametric curve in the complex plane given by

$$
\begin{aligned}
& x(v)=\left(r_{1}-r_{2}\right) \cos (v)+d \cos \left(\left(\frac{r_{1}}{r_{2}}-1\right) v\right), \\
& y(v)=\left(r_{1}-r_{2}\right) \sin (v)-d \sin \left(\left(\frac{r_{1}}{r_{2}}-1\right) v\right),
\end{aligned}
$$

$v \in \mathbb{R}$. To avoid remarks about degenerate cases we will assume that

$$
r_{1} \neq r_{2} \quad \text { and } \quad r_{1} r_{2} d \neq 0 .
$$

Recall that, for $r_{1}>r_{2}>0$ and $d>0$, the mechanical plane curve generated by a point $P$ attached to a circle $C_{2}$ with radius $r_{2}$ rolling about the inside of a fixed circle $C_{1}$ with radius $r_{1}$ is described by the above parametrization where $d$ denotes the distance from the point $P$ to the center of the circle $C\left(r_{2}\right)$ (see [Law]). Here, the notation $C(r)$ for $r>0$ means the circle $C(r)=\{z \in \mathbb{C},|z|=r\}$ endowed with the usual orientation. Observe that we do not make any restriction on the sign of the parameters $\left(r_{1}, r_{2}, d\right)$ of a trochoid. To construct the example we need to study that family of curves. Denote by $A\left(s_{1}, s_{2}\right) \subset \mathbb{C}$ the compact annulus with center at the origin and radii $s_{1}>s_{2}$, where

$$
\begin{aligned}
& s_{1}=\left|r_{1}-r_{2}\right|+|d|, \\
& s_{2}=|| r_{1}-r_{2}|-| d|| .
\end{aligned}
$$

The set $\Gamma=A\left(s_{1}, s_{2}\right) \times[-l, l] \subset \mathbb{C} \times \mathbb{R}$ will be called a cylindrical shell of revolution. We are going to construct a dense curve in $\Gamma$.

Lemma 2. Suppose that $\phi$ is an irrational number, $l>0$ and $\mu \in S^{1}$. The sequence

$$
\left(a_{k}, b_{k}\right)=\left(d \mu e^{-2 k \phi \pi i}, l e^{k \frac{\phi}{2} \pi i}\right), \quad k \in \mathbb{Z},
$$

is dense in an embedded circle of type $(p, q)=(-4,1)$ on the torus $C(|d|) \times C(l)$. 
Proof. Consider the function

$$
f:[0,4] \rightarrow C(|d|) \times C(|l|), \quad f(t)=\left(d \mu e^{-2 \pi t i}, l e^{\pi \frac{t}{2} i}\right)
$$

and the sequence $\left\{c_{k}\right\}$ in the domain of $f$ defined by

$$
c_{k} \equiv \phi k, \quad \bmod 4, \quad k \in \mathbb{Z} .
$$

Of course, this sequence is dense in the interval because $\phi$ is irrational. Since $f\left(c_{k}\right)=\left(a_{k}, b_{k}\right)$ and $f$ is a $C^{\infty}$ parametrization of an embedded circle of type $(-4,1)$, we conclude that the sequence image is dense in this embedded circle.

Lemma 3. Suppose that $l>0$. The parametric curve given by

$$
\begin{aligned}
& x(v)=\left(r_{1}-r_{2}\right) \cos (v)+d \cos \left(\left(\frac{r_{1}}{r_{2}}-1\right) v\right), \\
& y(v)=\left(r_{1}-r_{2}\right) \sin (v)-d \sin \left(\left(\frac{r_{1}}{r_{2}}-1\right) v\right), \\
& z(v)=l \sin \left(\frac{r_{1}}{2 r_{2}} v\right),
\end{aligned}
$$

$v \in \mathbb{R}$, is in the cylindrical shell of revolution $\Gamma=A\left(s_{1}, s_{2}\right) \times[-l, l] \subset \mathbb{C} \times \mathbb{R}$. If $\frac{r_{1}}{r_{2}} \in \mathbb{Q}$, then the curve is periodic; otherwise, it is dense in that cylindrical shell of revolution.

Proof. The $x y$-projection of the curve is a trochoid in $\mathbb{C}$ and it can be written as

$$
x(v)+i y(v)=\left(r_{1}-r_{2}\right) e^{i v}+d e^{-i\left(\frac{r_{1}}{r_{2}}-1\right) v} .
$$

So, from the triangular inequalities we obtain that

$$
|| r_{1}-r_{2}|-| d|| \leq|x(v)+i y(v)| \leq\left|r_{1}-r_{2}\right|+|d| .
$$

This shows that the trochoid is in the annulus $A\left(s_{1}, s_{2}\right)$ and it is immediate to conclude that the curve $(x(v)+i y(v), z(v))$ is in the cylindrical shell of revolution $\Gamma$. Now, if $\frac{r_{1}}{r_{2}}=\frac{p}{q} \in \mathbb{Q}$, where $p, q \in \mathbb{Z}$ and $q \neq 0$, one can see that the curve is periodic because

$$
(x(v+4 q \pi)+i y(v+4 q \pi), z(v+4 q \pi))=(x(v)+i y(v), z(v)) .
$$

Let us assume that $\frac{r_{1}}{r_{2}}$ is not rational.

First case: Suppose that $\left|r_{1}-r_{2}\right| \geq|d|$.

Given a point $\left(w_{0}, b\right) \in A\left(s_{1}, s_{2}\right) \times[-l, l]$, choose a point $z_{1}$ in the core circle $C\left(\left|r_{1}-r_{2}\right|\right) \subset A\left(s_{1}, s_{2}\right)$ whose distance from $w_{0}$ is $|d|$. If $0 \leq \theta=\arg \left(z_{1}\right)<2 \pi$, we have that $z_{1}=\left|r_{1}-r_{2}\right| e^{i v_{k}}$ where $v_{k}=\theta+2 k \pi$. So,

$$
\begin{aligned}
x\left(v_{k}\right)+i y\left(v_{k}\right) & =\left|r_{1}-r_{2}\right| e^{i v_{k}}+d e^{-\left(\frac{r_{1}}{r_{2}}-1\right) i v_{k}} \\
& =z_{1}+d e^{-\left(\frac{r_{1}}{r_{2}}-1\right)(\theta+2 k \pi) i} \\
& =z_{1}+d \mu e^{-2 \frac{r_{1}}{r_{2}} k \pi i},
\end{aligned}
$$

for some unit complex number $\mu$. Therefore the point

$$
\begin{aligned}
a_{k} & =x\left(v_{k}\right)+i y\left(v_{k}\right)-z_{1} \\
& =d \mu e^{\left(-2 \frac{r_{1}}{r_{2}} k \pi i\right)}
\end{aligned}
$$


and $w_{0}-z_{1}$ belong to the same circle $C(|d|)$, for each $k$. Now, take the sequence

$$
b_{k}=l e^{\left(\frac{r_{1}}{2 r_{2}} k \pi i\right)}
$$

in the circle $C(l)$. Since $\frac{r_{1}}{r_{2}} \notin \mathbb{Q}$, from Lemma 2 we conclude that the sequence $\left\{\left(a_{k}, b_{k}\right)\right\}$ is dense in a circle of type $(-4,1)$ of the torus $C(|d|) \times C(l)$. Let $\left(w_{0}-z_{1}, \beta_{0}\right)$ be a point in this embedded circle such that the $y$-projection $\Pi$ : $C(l) \rightarrow[-l, l]$ of $\beta_{0}$ is the given point $b \in[-l, l]$. It is possible to choose $\beta_{0}$ because the embedded circle is transverse to the fibers $C(|d|) \times\{\beta\}$. If $\left(a_{k_{i}}, b_{k_{i}}\right)$ is a subsequence which converges to $\left(w_{0}-z_{1}, \beta_{0}\right)$, then

$$
\left(x\left(v_{k_{i}}\right)+i y\left(v_{k_{i}}\right), z\left(v_{k_{i}}\right)\right)=\left(z_{1}+a_{k_{i}}, \Pi\left(b_{k_{i}}\right)\right)
$$

converges to $\left(w_{0}, b\right)$. This shows that the curve is dense in the cylindrical shell of revolution $\Gamma=A\left(s_{1}, s_{2}\right) \times[-l, l]$.

Second case: Suppose that $\left|r_{1}-r_{2}\right|<|d|$.

In this case, the core circle is $C(|d|) \subset A\left(s_{1}, s_{2}\right)$. Given a point $\left(w_{0}, b\right) \in A\left(s_{1}, s_{2}\right) \times$ $[-l, l]$, choose a point $z_{1}$ in the core circle whose distance from $w_{0}$ is $\left|r_{1}-r_{2}\right|$. If $0 \leq \theta=\arg \left(z_{1}\right)<2 \pi$, we have that $z_{1}=d e^{i v_{k}}$ where $v_{k}=\theta+2 k \pi$. So, we proceed in the same way as before to find a sequence in the trochoid which converges to $\left(w_{0}, b\right)$.

Proof (Theorem 2). Consider the map

$$
\chi: \mathbb{C} \rightarrow \mathbb{C} \times \mathbb{R}, \quad \chi(z)=(L(z)-\overline{H(z)}, h(z))
$$

where

$$
\begin{aligned}
L(z) & =\left(r_{1}-r_{2}\right) e^{z}, \\
H(z) & =-d e^{\left(\frac{r_{1}}{r_{2}}-1\right) z} \\
h(z) & =4\left(\frac{d}{r_{2}}\right)^{\frac{1}{2}}\left|\frac{r_{2}}{r_{1}}\right|\left|r_{1}-r_{2}\right| \operatorname{Re}\left(i e^{\frac{r_{1}}{2 r_{2}} z}\right) .
\end{aligned}
$$

As before, the parameters satisfy the conditions $r_{1} \neq r_{2}$ and $r_{1} r_{2} d \neq 0$; however, we now impose the extra restrictions

$$
r_{2}>0, \quad \frac{r_{1}}{r_{2}} \notin \mathbb{Q}, \quad \frac{r_{1}}{r_{2}}<1 \quad \text { and } \quad d=r_{2}-r_{1} .
$$

By using the identity

$$
\frac{\partial}{\partial z} \operatorname{Re}(P(z))=\frac{1}{2} P^{\prime}(z)
$$

for any analytic function $P(z)$, one can easily show that the map $\chi$ satisfies the hypothesis of Theorem 1 . So, it is a conformal minimal immersion of the plane. Fixed $u \in \mathbb{R}$, the $x y$-projection of the curve $v \mapsto \chi(u+i v)$ is a (nondegenerate) trochoid, namely,

$$
\begin{aligned}
& x(v)=\left(R_{1}(u)-R_{2}(u)\right) \cos (v)+D(u) \cos \left(\left(\frac{R_{1}(u)}{R_{2}(u)}-1\right) v\right), \\
& y(v)=\left(R_{1}(u)-R_{2}(u)\right) \sin (v)-D(u) \sin \left(\left(\frac{R_{1}(u)}{R_{2}(u)}-1\right) v\right),
\end{aligned}
$$


whose data is

$$
R_{1}(u)=r_{1} e^{u}, \quad R_{2}(u)=r_{2} e^{u} \quad \text { and } \quad D(u)=-d e^{\left(\frac{r_{1}}{r_{2}}-1\right) u} .
$$

The main property is that the trochoid obtained by projection satisfies the same irrationality condition; namely, for each $u$ we have

$$
\frac{R_{1}(u)}{R_{2}(u)}=\frac{r_{1}}{r_{2}} \notin \mathbb{Q} .
$$

From Lemma 3, we conclude that the path $v \mapsto \chi(u+i v)$ is dense in the vertical cylindrical shell of revolution $\Gamma_{u}=A\left(s_{1}(u), s_{2}(u)\right) \times[-l(u), l(u)]$, whose dimensions are (see (3.4), (3.5) and Lemma 3$)$

$$
\begin{aligned}
s_{1}(u) & =\left|r_{1}-r_{2}\right|\left(e^{u}+e^{\left(\frac{r_{1}}{r_{2}}-1\right) u}\right), \\
s_{2}(u) & =\left|r_{1}-r_{2}\right|\left|e^{u}-e^{\left.\left(\frac{r_{1}}{r_{2}}-1\right) u\right)}\right|, \\
l(u) & =4\left(\frac{d}{r_{2}}\right)^{\frac{1}{2}}\left|\frac{r_{2}}{r_{1}}\right|\left|r_{1}-r_{2}\right| e^{\frac{r_{1}}{2 r_{2}} u} .
\end{aligned}
$$

It is clear that the image of the map $\chi$ is dense in the region

$$
\Gamma=\bigcup_{u \in \mathbb{R}} \Gamma_{u} .
$$

The $x y$-plane is contained in the interior of $\Gamma$, since the height of the vertical cylindrical shell of revolution $\Gamma_{u}$ is $2 l(u)>0$, the smaller radius satisfies $s_{2}(0)=0$ and $s_{2}(u) \rightarrow+\infty$ when $u \rightarrow+\infty$. The region $\Gamma$ is not dense in $\mathbb{C} \times \mathbb{R}$ because there exist infinitely many cylinders which do not intersect $\Gamma$, for example, the cylinder

$$
R=A\left(0, s_{1}(1)\right) \times[49 l(1),+\infty) .
$$

The restrictions $\frac{r_{1}}{r_{2}}<1, d=r_{2}-r_{1}$ and the inequality $e^{u}+e^{\left(\frac{r_{1}}{r_{2}}-1\right) u} \geq 2$ allow us to write that

$$
\begin{aligned}
d s & =\left(\left|r_{1}-r_{2}\right| e^{u}+d e^{\left(\frac{r_{1}}{r_{2}}-1\right) u}\right)|d z| \\
& \geq 2\left|r_{1}-r_{2}\right||d z| .
\end{aligned}
$$

It is sufficient to show that the immersed plane is complete. To study the Gaussian curvature $K$, we observe that

$$
L^{\prime}(z)=k_{1} e^{z}, \quad H^{\prime}(z)=k_{2} e^{\left(\frac{r_{1}}{r_{2}}-1\right) z}, \quad \frac{g^{\prime}(z)}{g(z)}=k_{3}, \quad \text { and } \quad \frac{\partial h}{\partial z}=k_{4} e^{\frac{r_{1}}{2 r_{2}}}
$$

where $k_{i}, i=1,2,3,4$, are nonzero constants. From (2.8), we get

$$
K(u+i v)=-4\left[\frac{\left|k_{3} k_{4}\right|}{\left(\left|k_{1}\right| e^{\left(1-\frac{r_{1}}{4 r_{2}}\right) u}+\left|k_{2}\right| e^{\left(\frac{3 r_{1}}{4 r_{2}}-1\right) u}\right)^{2}}\right]^{2} .
$$

Since the expoents of $e^{\left(1-\frac{r_{1}}{4 r_{2}}\right) u}$ and $e^{\left(\frac{3 r_{1}}{4 r_{2}}-1\right) u}$ have opposite signs, we may easily conclude that $K$ is bounded. This completes the proof of Theorem 2. 


\section{REFERENCES}

[And] Andrade, P.; Enneper Immersions; Jorn. D'Ann. Math. vol LXXV, (1998), 121-134. CMP 99:04

[B-C] Barbosa, J. L. and Colares, A. G.; Minimal Surfaces in $\mathbb{R}^{3}$; Lectures Notes in Math. $n^{o}$ 1195, Springer-Verlag, 1986. MR 87j:53010

[Jor] Jorge, L. P.; personal conversation.

[J-S] Jenkins, H., Serrin, J.; Variational problems of minimal surfaces, type I; Arch. Rat. Anal. 12 (1963), 185-212. MR 26:2729

[Law] Lawrence, J. D.; A Catalog of Special Plane Curves, Dover Publications Inc. (1972).

[Rsb] Rosenberg, H.; A complete embedded minimal surface in $\mathbb{R}^{3}$ of bounded curvature is proper; preprint.

Universidade Federal do Ceará, Departamento de Matemática, Campus do Pici Bloco 914, CEP 60.455-760 Fortaleza, CE, Brazil

E-mail address: andrade@mat.ufc.br 